\title{
Equilibration of integer quantum Hall edge states
}

\author{
D. L. Kovrizhin and J. T. Chalker \\ Theoretical Physics, Oxford University, 1, Keble Road, Oxford OX1 3NP, United Kingdom \\ (Received 30 September 2010; revised manuscript received 29 June 2011; published 19 August 2011)
}

\begin{abstract}
We study equilibration of quantum Hall edge states at integer filling factors, motivated by experiments involving point contacts at finite bias. Idealizing the experimental situation and extending the notion of a quantum quench, we consider time evolution from an initial nonequilibrium state in a translationally invariant system. We show that electron interactions bring the system into a steady state at long times. Strikingly, this state is not a thermal one: Its properties depend on the full functional form of the initial electron distribution and not simply on the initial energy density. Further, we demonstrate that measurements of the tunneling density of states at long times can yield either an overestimate or an underestimate of the energy density, depending on details of the analysis, and discuss this finding in connection with an apparent energy loss observed experimentally. More specifically, we treat several separate cases: for filling factor $v=1$ we discuss relaxation due to finite-range or Coulomb interactions between electrons in the same channel, and for filling factor $v=2$ we examine relaxation due to contact interactions between electrons in different channels. In both instances we calculate analytically the long-time asymptotics of the single-particle correlation function. These results are supported by an exact solution at arbitrary time for the problem of relaxation at $v=2$ from an initial state in which the two channels have electron distributions that are both thermal but with unequal temperatures, for which we also examine the tunneling density of states.
\end{abstract}

DOI: 10.1103/PhysRevB.84.085105

PACS number(s): 71.10.Pm, 73.23.-b, 73.43.-f, 42.25.Hz

\section{INTRODUCTION}

Recent experiments have stimulated renewed interest in the process of equilibration for an isolated quantum system. Some of the most prominent of these studies have involved time evolution in cold atomic gases, after a sudden change in system parameters. ${ }^{1,2}$ Other measurements, remarkably, are able to probe coherent quantum dynamics in conventional condensed matter systems far from equilibrium. ${ }^{3-8}$ Theoretical work has included studies of general features of dynamics after a quantum quench, ${ }^{9}$ and of the approach to thermal equilibrium, ${ }^{10}$ as well as detailed investigations of individual model systems. ${ }^{11,12}$

A notable result from experiments on cold atomic gases is the absence of relaxation in some cases, despite there being strong interactions between particles. ${ }^{1}$ This has been attributed to the fact that the system concerned is, to a good approximation, described by the Hamiltonian for an integrable model.

In the condensed matter setting, a series of recent investigations have examined aspects of the physics of quantum Hall edge states far from equilibrium. These include: the discovery of interaction effects in electronic Mach-Zehnder interferometers ${ }^{3}$ the detection of thermal transport by quantum Hall edge states; ${ }^{4}$ and the measurement ${ }^{5}$ of the energy distribution of electrons in an edge state driven out of equilibrium by passage through a quantum point contact (QPC) at finite bias voltage, and the observation ${ }^{6}$ and control $^{7}$ of the relaxation of this distribution.

The theoretical work we describe in this paper is motivated in part by the last of these sets of experiments. In outline, the experiments ${ }^{5-7}$ involve bringing together, at a QPC, integer quantum Hall edge states that originate from contacts at different potentials. Tunneling between edges within the QPC generates a nonequilibrium electron energy distribution downstream from the contact. This distribution is measured ${ }^{5}$ using a quantum dot as a spectrometer, as a function of propagation distance. ${ }^{6}$ It relaxes to a stationary form, which is close to thermal but with an effective temperature higher than that of the sample as a whole. The fact that this distribution propagates further with little change suggests that electrons in edge states are weakly coupled to other degrees of freedom.

These observations raise some important theoretical questions. A central one stems from the fact that the usual, chiral Luttinger liquid model for quantum Hall edge states ${ }^{13}$ is integrable, being simply quadratic in the edge magnetoplasmon coordinates. Within this description, the number of excitation quanta in each collective mode is conserved under time evolution. In this context, it is natural to ask how relaxation takes place in edge states, and what steady state is reached at long times.

The main difficulty in addressing these issues arises because interactions are simplest to handle using bosonization, while a QPC is most naturally represented using fermionic variables. The two existing theoretical discussions ${ }^{14,15}$ of edge state relaxation aim at a faithful, but necessarily approximate, treatment of the experimental arrangement, including the QPC. Our objective in this paper is to explore a complementary direction. We study interaction effects on time evolution in a translationally invariant system, omitting the QPC altogether from our model Hamiltonian. In its place, we choose the initial state of the system to have an electron distribution in momentum like that generated when noninteracting fermions pass through a tunneling contact at finite bias voltage. Our problem, therefore, has the features of a quantum quench, ${ }^{9}$ except that the initial state is not chosen to be the ground state of a simple Hamiltonian. In our treatment we expect evolution as a function of time to correspond qualitatively to evolution in the experimental system as a function of distance from the QPC, with time and distance in the two pictures related by a characteristic edge state velocity. While 
this correspondence is not precise, our exact treatment of interactions brings advantages. In particular, we are able to show that the one-electron correlation function reaches a stationary form in the long-time limit that is not thermal. It would probably be hard to know whether this conclusion was reliable if it were the result of an approximate calculation. Our calculations draw on theoretical ideas developed in recent discussions ${ }^{16-18}$ of nonequilibrium effects in Mach-Zehnder interferometers.

The remainder of the paper is organized as follows. In Sec. II we give an overview of the results and discuss the relation of our approach to experiments and to previous theory. In Sec. III we introduce the model and notation we use. In Sec. IV we show how plasmon dispersion arising from finite range or Coulomb interactions in an edge at $v=1$ generates a stationary electron distribution at long times for an initial state that has no many-particle correlations but it is otherwise general. We then specialize this treatment to an initial state in which the electron distribution in momentum consists of a double step. In Sec. V we treat a system at $v=2$ with contact interactions between electrons in different channels. We obtain an exact solution for the time evolution with edges initially at different temperatures and study the long-time distribution reached for a general initial state. Finally, in Sec. VI we use this solved example to discuss the tunneling density of states and implications for experimental estimates of the energy density in the steady state.

\section{OVERVIEW OF RESULTS}

In the experiments ${ }^{6}$ that we are concerned with, edge channels of a quantum Hall system at filling factor $v=2$ are driven out of equilibrium by applying bias voltage $V$ to a QPC. Edges that originate from contacts with different potentials meet at the QPC where, depending on its width, either the inner or the outer channels of the two edges are coupled by quantum tunneling. As a result, immediately after the QPC, the electrons in these channels have a nonequilibrium energy distribution. For example, in a noninteracting system at zero temperature, this distribution has two steps with energy separation $\mathrm{eV}$, as shown in Fig. 1. The energy distribution of electrons is expected to evolve as a function of distance downstream from the QPC, because of inelastic scattering induced by interactions. This distribution is measured in the outer channel at successive distances from the QPC, using a quantum dot with a single active electronic level. It is found that the double-step distribution generated by the QPC equilibrates after a propagation length of order $10 \mu \mathrm{m}$. The observed equilibrium distribution is similar to a Fermi function, with an effective temperature close to that set by the average electron energy density in the channels just after the QPC.

Two different approaches have been used in previous work to model these experiments. One ${ }^{14}$ is formulated in terms of electron operators, using a Boltzmann-like equation to study the evolution of the electron distribution with distance as a consequence of electron-electron interactions. This method has the advantage that the initial electron distribution can be calculated simply by using a single-particle treatment of the QPC. Its limitation stems from the fact that the integrability of the usual, chiral Luttinger liquid model for edge states is

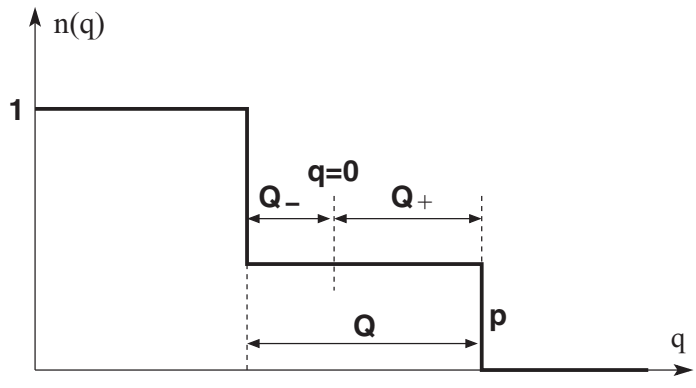

FIG. 1. Double step electron momentum distribution. The width $Q$ of the step represents a voltage $\hbar v Q$ applied to a QPC, which has tunneling probability $p$. The quantities $Q_{+}$and $Q_{-}$are determined following Eq. (32).

presumably not respected. The other approach ${ }^{15}$ is formulated in terms of edge magnetoplasmons. This allows a detailed treatment of interactions, at the cost of a phenomenological treatment of the QPC, in which the plasmon distribution just after the QPC is estimated from shot noise in the charge.

We evade some of these difficulties by treating relaxation of an initial distribution in a translation-invariant edge. The relaxation is caused by scattering of electrons between different single-particle momentum eigenstates. By contrast, the collective plasmon modes of the system do not relax, because within standard approximations the bosonized Hamiltonian is quadratic. Instead, using the language of plasmons, relaxation occurs because in the interacting system different modes have different group velocities. To expand on this viewpoint, we use it to estimate the relaxation time for an initial state in which electrons occupy single-particle levels independently, with a specified energy distribution. This state can be viewed in terms of particle and hole excitations above a Fermi sea. It is characterized by the mean separation $L_{\text {init }}$ between these excitations. For an electron energy distribution having two steps at energy separation $\mathrm{eV}$, with edge velocity $v$, we have $L_{\text {init }} \sim \hbar v / \mathrm{eV}$. Because of plasmon dispersion, the width $\ell$ of particle and hole wave packets broadens with time $t$ : the relaxation time $\tau$ is the time scale at which their width is comparable to their separation.

The simplest application of these ideas is provided by a system at filling factor $v=2$ with contact interactions between electrons in different channels. Plasmon dispersion in this situation is characterized by velocities $v_{-}$and $v_{+}$for slow modes and fast modes, as reviewed in Sec. V. Then the width of the wave packet grows as $\ell \sim\left(v_{+}-v_{-}\right) t$ and the relaxation time is

$$
\tau=\frac{v_{+}+v_{-}}{v_{+}-v_{-}} \frac{\hbar}{\mathrm{eV}} .
$$

Note that this time scale and the corresponding length scale (similar to one identified ${ }^{19}$ in Ref. 15) vary inversely with bias voltage V. It would be interesting to search for such a dependence in experiment: this might be done from the data in Fig. 2 of the Supplementary Material to Ref. 6, by extracting the decay length of $T_{\text {exc }}$ and analyzing its dependence on $\delta V_{D}$.

As a second example, consider a single edge channel at filling factor $v=1$ with finite range interactions. The plasmon 


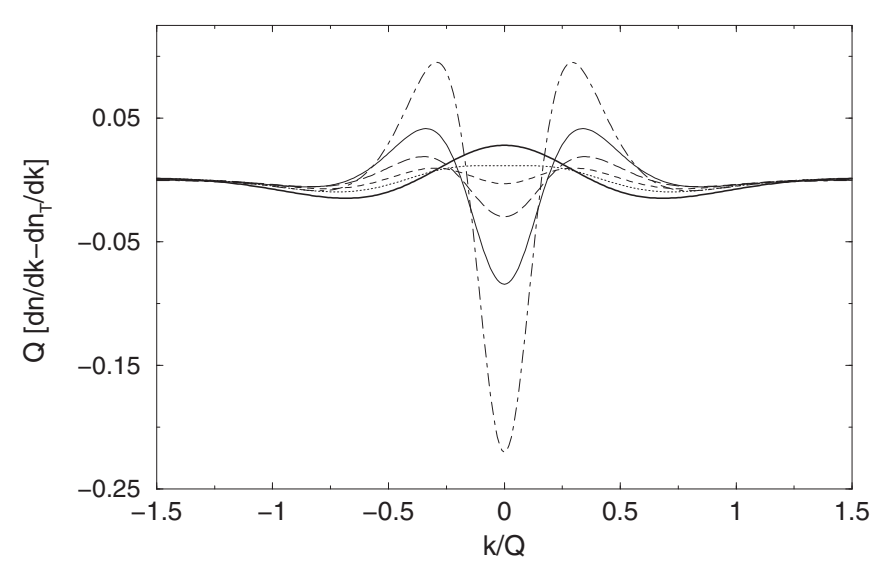

FIG. 2. Difference between derivatives of the electron momentum distribution functions in an steady state described by Eq. (45) and in a thermal state with the same energy density; (thick solid line) $p=0.5$; (dotted line) $p=0.3$; (short-dashed line) $p=0.25$; (long-dashed line) $p=0.2$; (thin solid line) $p=0.15$; (dot-dashed line) $p=0.1$.

frequency $\omega_{q}$ varies with wave vector $q$, having the small- $q$ expansion

$$
\omega_{q}=v q-(v / b)(b q)^{3} / 3 \cdots,
$$

where $b>0$ is a length that characterizes the range and strength of interactions. In place of $v_{+}-v_{-}$we take the difference between the group velocity $\partial_{q} \omega_{q}$ at $q=0$ and at $q=\ell^{-1}$, which is $v(b / \ell)^{2}$. Setting $\ell=v t(b / \ell)^{2}$ yields $\ell=b(v t / b)^{1 / 3}$ and from $\ell=L_{\text {init }}$ we obtain

$$
\tau=\left(\frac{v}{b}\right)^{2}\left(\frac{\hbar}{\mathrm{eV}}\right)^{3}
$$

Thus at $v=1$ with finite range interactions, we find a time scale and length scale that vary with bias voltage as $V^{-3}$, in striking contrast to behavior at $v=2$. Again, it would be of interest to test this in experiment.

For unscreened Coulomb interactions the plasmon dispersion is

$$
\omega_{q}=q[v+u \ln (1 / b q)]
$$

and the width of the electron wave packet grows with time as $\ell=u t$, giving

$$
\tau=\frac{\hbar u}{e v V} .
$$

Similar scaling relations have been discussed in Ref. 16 in the context of the decoherence in electronic Mach-Zehnder interferometers.

We show in the remainder of this paper that at times much longer than $\tau$, the equal-time, one-electron correlation function reaches a stationary form. For $v=1$ and an initial state in which single-particle levels are occupied independently, we determine this form exactly as a functional of the initial distribution. Its short-distance behavior is fixed by the energy density in the nonequilibrium state and characterized by an effective temperature. Its behavior at long distances, however, depends on the full functional form of the initial distribution. We present detailed results for the case of a double-step initial distribution, as produced at a QPC. For this example we also consider the electron momentum distribution at long times, which shows power-law behavior at small momenta, with an exponent that depends on tunneling probability of the QPC. At large momenta the distribution is close to thermal with an effective temperature given by the excess energy.

The electron distribution is probed in the experiments of Refs. 5-7 and 20 by measurement of the tunneling current through a quantum dot that has a single active energy level weakly coupled to the edge state. Without interactions, this current is proportional to the occupation probability of edgestate orbitals at the energy level of the dot and so yields a straightforward determination of the electron distribution in energy. With interactions, however, the situation is more complicated, since matrix elements for tunneling between the edge and the dot depend on the many-body state of the quantum Hall edge. We examine the consequences of interaction effects for the interpretation of tunneling current measurements in Sec. VI, focusing in particular on estimates of the energy density. We find that the energy density can be overestimated or underestimated, depending on how such measurements are analyzed.

\section{MODEL AND OBSERVABLES}

In the following we study edge states in the integer quantum Hall regime at filling factors $v=1$ and $v=2$. The microscopic Hamiltonian $\hat{H}$ is a sum

$$
\hat{H}=\hat{H}_{\text {kin }}+\hat{H}_{\text {int }}
$$

of contributions from kinetic energy $\hat{H}_{\text {kin }}$ and interaction energy $\hat{H}_{\text {int }}$. For $n_{c}$ chiral copropagating electron channels, with channel index $\eta$ and Fermi velocities $v_{\eta}$, we have

$$
\hat{H}_{\mathrm{kin}}=-i \hbar \sum_{\eta=1}^{n_{c}} v_{\eta} \int_{-L / 2}^{L / 2} \hat{\psi}_{\eta}^{+}(x) \partial_{x} \hat{\psi}_{\eta}(x) d x .
$$

Here we impose periodic boundary conditions on a system of length $L$ so that allowed wave vectors are $k=2 \pi n / L$, with integer $n$. Fermionic creation and annihilation operators $\hat{c}_{k \eta}^{+}$ and $\hat{c}_{k \eta}$ for an electron with wave vector $k$ on the edge $\eta$ obey standard anticommutation relations $\left\{\hat{c}_{k \eta}, \hat{c}_{p \eta^{\prime}}^{+}\right\}=\delta_{k p} \delta_{\eta \eta^{\prime}}$. The field operator $\hat{\psi}_{\eta}(x)$, which annihilates an electron at position $x$ on the channel $\eta$, is

$$
\hat{\psi}_{\eta}(x)=\frac{1}{\sqrt{L}} \sum_{k=-\infty}^{\infty} \hat{c}_{k \eta} e^{i k x} .
$$

We consider translationally invariant interactions described by the potential $U_{\eta \eta^{\prime}}\left(x-x^{\prime}\right)$ so

$$
\hat{H}_{\mathrm{int}}=\frac{1}{2} \int_{-L / 2}^{L / 2} \int_{-L / 2}^{L / 2} d x d x^{\prime} U_{\eta \eta^{\prime}}\left(x-x^{\prime}\right) \hat{\rho}_{\eta}(x) \hat{\rho}_{\eta^{\prime}}\left(x^{\prime}\right) \text {. }
$$

We discuss two different cases: a single edge channel at $v=1$, with finite range interactions, and a two-channel system at $v=2$, with contact interactions between electrons in different channels. Contact interactions between electrons in the same channel can be absorbed into a renormalization of Fermi velocities.

Our first objective is to obtain the equal time singleparticle correlation function. Using a time argument to indicate 
operators $\hat{\psi}_{\eta}(x, t)=e^{i \hat{H} t} \hat{\psi}_{\eta}(x) e^{-i \hat{H} t}$ in their Heisenberg representation, we write this quantity at time $t$ as

$$
G_{\eta}\left(x-x^{\prime}, t\right)=\left\langle\hat{\psi}_{\eta}^{+}(x, t) \hat{\psi}_{\eta}\left(x^{\prime}, t\right)\right\rangle,
$$

where the average is calculated with respect to the initial state, yet to be specified. The electron momentum distribution in a translationally invariant system is then

$$
n_{\eta}(k, t)=\int_{-L / 2}^{L / 2} d x e^{i k x} G_{\eta}(x, t) .
$$

In thermal equilibrium at inverse temperature $\beta$ the singleparticle correlation function has at $v=1$ the form

$$
G(x, t)=i\{2 \beta \hbar v \sinh [\pi(x-v t+i a) / \beta \hbar v]\}^{-1}
$$

with a length scale $\beta \hbar v$, time scale $\beta \hbar$ and a short-distance cutoff $a$.

The experiments of Refs. 5-7 measure the current through a quantum dot (QD) weakly coupled to one edge channel. Assuming sequential incoherent tunneling through a QD with a single energy level $E$, perturbation theory in the tunneling amplitude $t_{D}$ gives for this current the expression ${ }^{21}$

$$
\frac{e\left|t_{D}\right|^{2}}{\hbar} v_{\eta}(E)
$$

where the tunneling density of states is at long times

$$
v_{\eta}(E)=\lim _{t \rightarrow \infty} \hbar^{-1} \int_{-\infty}^{\infty} d \tau\left\langle\hat{\psi}^{+}(0, t+\tau) \hat{\psi}(0, t)\right\rangle e^{-i E \tau / \hbar} .
$$

\section{EQUILIBRATION AT $\boldsymbol{v}=1$}

To compute the time evolution of operators under $\hat{H}$ we use bosonization, ${ }^{22}$ employing the same notation as in Sec. IV C of Ref. 18. Consider a system at $v=1$. The Hamiltonian in bosonic form is

$$
\hat{H}=\sum_{q>0} \hbar \omega_{q} \hat{b}_{q}^{+} \hat{b}_{q}
$$

where $\hat{b}_{q}^{+}$is an operator creating a plasmon with wave vector $q$ and energy $\hbar \omega_{q}$, and we omit the channel label $\eta$. Let the Fourier transform of the interaction potential be

$$
u(q)=\int_{-\infty}^{\infty} d x e^{-i q x} U(x) .
$$

The boson dispersion relation is then

$$
\omega_{q}=v q[1+u(q) / 2 \pi \hbar v] .
$$

In this section we express the correlation function, Eq. (10), in terms of the electron density operator $\hat{\rho}(x)=\hat{\psi}^{+}(x) \hat{\psi}(x)$ written in the Schrödinger representation. The result, Eq. (19), involves an average of $\hat{\rho}(y)$ with a kernel, denoted below by $K(x, t ; y)$. Quantum fluctuations of this average are Gaussian at long times, because the range of the kernel becomes much larger than the correlation length in the initial state. For this reason, the correlation function in the long-time limit can be expressed as a functional of the second moment of the density in the initial state. More formally, one can write a cumulant expansion for the correlation function, in which the second-order cumulant is time independent and higher-order contributions decay to zero at long times if the plasmon dispersion $\omega_{q}$ is not exactly linear in $q$.

\section{A. Correlations at $v=1$ in terms of initial density operator}

Introduction of the bosonic field

$$
\hat{\phi}(x, t)=-\sum_{q>0}(2 \pi / q L)^{1 / 2}\left(\hat{b}_{q} e^{i q x-i \omega_{q} t}+\text { H.c. }\right) e^{-q a / 2}
$$

allows the electron correlation function to be written as

$$
G(x, t)=(2 \pi a)^{-1}\left\langle e^{i \hat{\phi}(x, t)} e^{-i \hat{\phi}(0, t)}\right\rangle .
$$

In order to evaluate this average, we express the boson operators $\hat{b}_{q}$ and $\hat{b}_{q}^{+}$in terms of $\hat{\rho}(x)$. From the standard expression

$$
\hat{\rho}(y)=\frac{1}{2 \pi} \sum_{q>0}(2 \pi / q L)^{1 / 2}\left[i q \hat{b}_{q} e^{i q y}-i q \hat{b}_{q}^{+} e^{-i q y}\right],
$$

we have for $k>0$

$$
\int_{-L / 2}^{L / 2} \hat{\rho}(y) e^{-i k y} d y=\frac{L}{2 \pi}\left(\frac{2 \pi}{k L}\right)^{1 / 2} i k \hat{b}_{k} .
$$

Thus the difference in bosonic fields

$$
\hat{\phi}(x, t)-\hat{\phi}(0, t)=\int_{-L / 2}^{L / 2} K(x, t ; y) \hat{\rho}(y) d y,
$$

is given by a spatial average of the electron density operator weighted with the kernel

$$
K(x, t ; y)=-i \sum_{q \neq 0} \frac{2 \pi}{q L}\left(e^{-i q x}-1\right) e^{i \omega_{q} t+i q y} .
$$

This yields an expression for the correlation function in terms of fermionic operators

$$
G(x, t)=(2 \pi a)^{-1} e^{I(x, a) / 2}\left\langle e^{i \int d y K(x, t ; y) \hat{\rho}(y)}\right\rangle,
$$

where

$$
I(x, a) \equiv[\hat{\phi}(x, t), \hat{\phi}(0, t)]=\ln [(a+i x) /(a-i x)] .
$$

\section{Long-time asymptotics of the kernel}

Considered as a function of $y$, the kernel $K(x, t ; y)$ at time $t=0$ is a step of width $|x|$ with height $-2 \pi$. In the noninteracting system for $t>0$, this step propagates at velocity $v$ without change in shape. With interactions, the relaxation time of $\tau$ introduced in Sec. II is the time scale for the step to change shape. We are interested in behavior of $K$ at long times in the presence of interactions. Consider first finite-range interactions with the plasmon dispersion at small wave vector given by Eq. (2). We separate out the average motion by writing $y=x / 2-v t+\lambda$. This gives for the phase appearing in Eq. (18)

$$
q(y-x / 2)+\omega_{q} t=q \lambda-(v t / b)(b q)^{3} / 3+\cdots
$$


at small $q$. Defining a length scale $l_{S}=b(v t / b)^{1 / 3}$ as in Sec. II, the kernel at long times has the form

$$
K(x, t, y)=-2 \int_{-\infty}^{\infty} \frac{d Q}{Q} \sin \frac{Q x}{2 l_{S}} \cos \left[Q\left(\lambda / l_{S}\right)+Q^{3} / 3\right] .
$$

Explicit evaluation for $|x| \ll l_{S}$ yields

$$
K(x, t, y)=-2 \pi\left(x / l_{S}\right) \operatorname{Ai}\left[\lambda / l_{S}\right] .
$$

Thus the range of the kernel grows with time as $t^{1 / 3}$ and its amplitude decays as $t^{-1 / 3}$.

We can obtain asymptotics of the kernel for a system with Coulomb interactions in a similar way. In this case the plasmon dispersion is given by Eq. (4). Writing $y=$ $x / 2-v t-u t \ln (u t / b)+\xi$, the phase appearing in Eq. (18) is

$$
q(y-x / 2)+\omega_{q} t=q \lambda+q u t \ln (1 / q u t) .
$$

Defining $l_{C}=u t$, we have

$$
K(x, t, y)=-2 \int_{-\infty}^{\infty} \frac{d Q}{Q} \sin \frac{Q x}{2 l_{C}} \cos \left(Q \lambda / l_{C}-Q \ln |Q|\right) .
$$

Hence, with Coulomb interactions at long times, the range of the kernel grows as $t$ and its amplitude decays as $t^{-1}$.

\section{Cumulant expansion}

To evaluate the average in Eq. (19) we write it in terms of cumulants as

$$
\begin{aligned}
\left\langle e^{i \int K(x, t ; y) \hat{\rho}(y) d y}\right\rangle= & \exp \left[\sum_{n} \frac{i^{n}}{n !} \int_{-L / 2}^{L / 2} d y d z_{1} \cdots d z_{n-1}\right. \\
& \left.\times F_{n}\left(x, t, y ; z_{1} \cdots z_{n-1}\right) C_{n}\left(z_{1} \cdots z_{n-1}\right)\right],
\end{aligned}
$$

where the $n$-th order cumulant of the electron density $\hat{\rho}(y)$ is

$$
C_{n}\left(z_{1}, \ldots, z_{n-1}\right)=\left\langle\rho(y) \rho\left(y+z_{1}\right) \cdots \rho\left(y+z_{n-1}\right)\right\rangle
$$

and the $n$-th order convolution of kernels is

$$
\begin{aligned}
F_{n}\left(x, t ; z_{1} \cdots z_{n-1}\right)= & \frac{1}{2 \pi} \int_{-L / 2}^{L / 2} d y K(x, t ; y) \\
& \times K\left(x, t ; y+z_{1}\right) \cdots K\left(x, t ; y+z_{n-1}\right) .
\end{aligned}
$$

Consider the second-order contribution. This is

$$
\begin{aligned}
F_{2}(x, t ; z)= & \frac{1}{2 \pi} \int_{-L / 2}^{L / 2} d y K(x, t ; y) K(x, t ; y+z) \\
= & \frac{1}{2 \pi}(-i)^{2}(2 \pi / L)^{2} \sum_{q, k \neq 0} \frac{1}{q k}\left(e^{-i q x}-1\right)\left(e^{-i k x}-1\right) \\
& \times \int_{-L / 2}^{L / 2} d y e^{i(q+k) y} e^{i k z} e^{i\left(\omega_{q}+\omega_{k}\right) t} .
\end{aligned}
$$

The result, independent of both time and interactions, is

$$
F_{2}(x, z)=\pi(|x+z|+|x-z|-2|z|) .
$$

It is a triangle as a function of $z$, which is nonzero in the region $(-x, x)$ and has amplitude $F_{2}(x, 0,0)=2 \pi|x|$.

At third order we have

$$
\begin{aligned}
F_{3}\left(x, t ; z_{1}, z_{2}\right)= & -(-i)^{3} \int_{-\infty}^{\infty} \frac{d q}{q} \int_{-\infty}^{\infty} \frac{d p}{p} \frac{1}{p+q} \\
& \times\left(e^{-i q x}-1\right)\left(e^{-i p x}-1\right)\left(e^{i(p+q) x}-1\right) \\
& \times e^{i\left(\omega_{p}+\omega_{q}-\omega_{p+q}\right) t} e^{i p z_{1}} e^{-i(p+q) z_{2}} .
\end{aligned}
$$

Time enters the integrand of this expression through the factor $e^{i\left(\omega_{p}+\omega_{q}-\omega_{p+q}\right) t}$. Without interactions $\omega_{q}+\omega_{p}-\omega_{p+q}=0$, but in an interacting system, this factor oscillates rapidly at long times as a function of $p$ and $q$. For large $t$ the integrals are therefore small and are dominated by low-frequency contributions. In this regime we can use the expansion $\omega_{q}+\omega_{p}-\omega_{p+q} \propto q^{3}+p^{3}-[p+q]^{3}$ and remove the time dependence from the exponent by rescaling variables, with $q=Q / l_{S}, p=P / l_{S}$, as in the discussion of the kernel, and $x=X l_{S}, z_{1,2}=Z_{1,2} l_{S}$. In this way we find $F_{3}=t^{-1 / 3} \times(\mathrm{a}$ time-independent function of $X, Z_{1}, Z_{2}$ ). Because the functions $C_{n}\left(z_{1} \cdots z_{n-1}\right)$ decay at large $z$ we can use in Eq. (26) the function $F_{3}$ evaluated at $Z_{1}=Z_{2}=0$, obtaining a contribution that decays as $t^{-1 / 3}$. An equivalent result holds for all higher-order cumulants. Similar arguments also apply in the case of Coulomb interactions, but with third- and higher-order terms decaying in time as $t^{-1}$.

The significance of these results is that contributions from all cumulants beyond the second one decay with time and may be neglected in the long-time limit. Cancellation of the high-order cumulants is known for thermal states as the Dzyaloshinskii-Larkin theorem ${ }^{12,23}$ but is not in general valid for nonequilibrium states. We have thus shown that a similar result holds in the long-time limit.

In summary, we obtain for the correlation function in the long-time limit the expression

$$
\begin{aligned}
\mathcal{G}(x)= & \lim _{t \rightarrow \infty} G(x, t)=(2 \pi a)^{-1} e^{\frac{1}{2} I(a)} \\
& \times \exp \left[-\pi \int_{-\infty}^{\infty} d z F_{2}(x, z)\langle\hat{\rho}(x) \hat{\rho}(x+z)\rangle\right] .
\end{aligned}
$$

This is one of the main results of this paper. In order to use it, we need to calculate $\langle\hat{\rho}(y) \hat{\rho}(y+z)\rangle$ for the initial state, which we do below.

\section{B. Long-time correlations}

As a check and because we shall need to refer to the results, we start by considering averages in the ground state, denoted by $\langle\cdots\rangle_{0}$. We require $\langle\hat{\rho}(0) \hat{\rho}(z)\rangle_{0}$ and find

$$
\langle\hat{\rho}(0) \hat{\rho}(z)\rangle_{0}=\frac{1}{(2 \pi)^{2}} \frac{1}{(a+i z)^{2}} .
$$

Integrating this with the function $F_{2}(x, z)$ [Eq. (29)] we have

$$
\int_{-\infty}^{\infty} d z F_{2}(x, z)\langle\hat{\rho}(x) \hat{\rho}(x+z)\rangle_{0}=-\frac{1}{2 \pi}\left[\ln a^{2}-\ln \left(x^{2}+a^{2}\right)\right] .
$$


Substituting Eqs. (31) and (20) into Eq. (30) we obtain for the ground-state correlation function

$$
\begin{aligned}
G(x, 0)= & (2 \pi a)^{-1} \exp \left\{\frac{1}{2}\left[\ln a^{2}-\ln \left(x^{2}+a^{2}\right)\right]\right\} \\
& \times[(a+i x) /(a-i x)]^{1 / 2}=\frac{i}{2 \pi} \frac{1}{x+i a},
\end{aligned}
$$

in agreement with Eq. (12), evaluated for $\beta \rightarrow \infty$.

Now consider an initial state in which single-particle momentum eigenstates are occupied independently with probability $n(k)$ as a function of wave vector $k$. We separate this probability into its value $n_{0}(k)$ for a filled Fermi sea with Fermi wave vector zero, and a deviation $\delta n(k)$, writing $n(k)=n_{0}(k)+\delta n(k)$. Without loss of generality, we take

$$
\int_{-\infty}^{\infty} \delta n(k) d k=0
$$

In this state we have

$$
\begin{aligned}
C_{2}(z) & =\frac{1}{L^{2}} \sum_{k_{1} k_{2} k_{3}}\left\langle\hat{c}_{k_{1}}^{+} \hat{c}_{k_{2}} \hat{c}_{k_{3}}^{+} \hat{c}_{k_{1}+k_{3}-k_{2}}\right\rangle e^{i\left(k_{1}-k_{2}\right) z} \\
& =\langle\hat{\rho}(0) \hat{\rho}(z)\rangle_{0}+C_{2}^{(1)}(z)+C_{2}^{(2)}(z),
\end{aligned}
$$

where we have introduced

$$
C_{2}^{(1)}(z)=\frac{1}{2 \pi^{2} z} \int_{-\infty}^{\infty} d q \delta n(q) \sin q z
$$

and

$$
C_{2}^{(2)}(z)=-\left|\frac{1}{2 \pi} \int d q \delta n(q) e^{-i q z}\right|^{2} .
$$

So in the long-time limit we have

$$
\mathcal{G}(x)=\frac{i}{2 \pi} \frac{1}{x+i a} \exp [-f(x)]
$$

with

$$
f(x)=\pi \int_{-\infty}^{\infty} d z F_{2}(x, z)\left[C_{2}^{(1)}(z)+C_{2}^{(2)}(z)\right] .
$$

This represents a second key result of our work, and we next analyze the behavior of $f(x)$.

\section{Short-distance asymptotics}

Consider $f(x)$ for $x$ much smaller than the quasiparticle spacing in the initial state. Since $F(x, z)$ is nonzero only for $|z|<|x|$ we require the small $z$ behavior of $C_{2}^{(1)}(z)$ and $C_{2}^{(2)}(z)$. Both can be expressed in terms of the energy density in the initial state, characterized by an effective temperature $T^{*}$. This is defined following the relation

$$
\frac{\hbar v_{F}}{2 \pi} \int \delta n(q) q d q=\frac{\pi}{12}\left(k_{B} T\right)^{2}
$$

between energy density and temperature $T$ for a thermal state. We have $C_{2}^{(1)}(z)=\left(k_{B} T^{*} / \hbar v_{F}\right)^{2} / 12+O\left(z^{2}\right)$ and $C_{2}^{(2)}(z)=$ $-\left[\pi z C_{2}^{(1)}\right]^{2}$. In this way we find

$$
f(x)=\frac{\pi^{2}}{6}\left(\frac{k_{B} T^{*}}{\hbar v_{F}}\right)^{2} x^{2}+O\left(x^{4}\right) .
$$

In summary, the short-distance behavior of the steady state is identical to that in thermal equilibrium at a temperature fixed by the initial energy density.

\section{Long-distance asymptotics}

At large $x$, the function $F_{2}(x, z)$ has slow dependence on $z$, but $C_{2}^{(1)}(z)$ and $C_{2}^{(2)}(z)$ fall to zero for $|z|$ much greater than the quasiparticle spacing in the initial state. We can therefore make the replacement $F_{2}(x, z)=2 \pi|x|$ in Eq. (37), obtaining

$$
f(x)=\pi|x| \int_{-\infty}^{\infty} d q\left[\operatorname{sgn}(q) \delta n(q)-|\delta n(q)|^{2}\right]+O\left(|x|^{0}\right) .
$$

The long-distance decay of the correlation function is therefore not in general determined solely by the energy density but instead depends on the entire momentum distribution in the initial state. In the following subsection we examine the consequences of these general results for a specific choice of initial distribution.

\section{Equilibration from double-step initial momentum distribution}

We discuss in detail the long-time correlations for an initial state that has a double-step momentum distribution as shown in the Fig. 1, which serves as a model for the electron distribution generated just after a QPC in tunneling experiments. ${ }^{5,6}$ For a QPC with a tunneling probability $p$ at bias voltage $V$, steps in $\delta n(q)$ are located at $q=-Q_{-}$and $q=Q_{+}$, with separation $Q_{+}+Q_{-} \equiv Q=\mathrm{eV} / \hbar v_{F}$, so

$$
\delta n(q)=\left\{\begin{array}{cc}
-(1-p) & -Q_{-}<q<0 \\
p & 0<q<Q_{+}
\end{array}\right.
$$

and $\delta n(q)=0$ if $q$ lies outside this range. Equation (32) implies $(1-p) Q_{-}=p Q_{+}$.

With these definitions we have an effective temperature

$$
T^{*}=\sqrt{3}\left(\hbar v_{F} / \pi k_{B}\right) Q \sqrt{p(1-p)} .
$$

It is interesting to examine for this initial distribution how the steady-state correlation function differs at long distances from a thermal one with the same effective temperature. We have

$$
\int_{-\infty}^{\infty} d q|\delta n(q)|^{2}=Q p(1-p)
$$

and

$$
\int_{-\infty}^{\infty} d q \operatorname{sgn}(q) \delta n(q)=2 Q p(1-p)
$$

so for $Q|x| \gg 1$

$$
f(x)=\pi p(1-p) Q|x| .
$$

For comparison, in a thermal state one has from Eq. (12)

$$
f(x)=\frac{k_{B} T}{\hbar v_{F}}|x| \text {. }
$$

A simple replacement of $T$ with $T^{*}$, expressed in terms of $p$ and $Q$ using Eq. (41), would lead one to expect in place of Eq. (42) the result (also for $Q|x| \gg 1$ )

$$
f(x)=\frac{\sqrt{3 p(1-p)}}{\pi} Q|x| .
$$


Clearly [except for the special value $p(1-p)=3 \pi^{-4}$ ], these are distinct forms. The difference is most pronounced if $p(1-p) \ll 1$, when the correlation function in the steady state decays much more rapidly with $|x|$ than would be the case in a thermal state with the same energy density.

For this case of a double-step initial distribution, the steady-state correlation function for general $x$ can be obtained explicitly in the form

$$
\mathcal{G}(x)=\frac{i}{2 \pi} \frac{1}{x+i a} \exp [-p(1-p) R(Q x)],
$$

where we have defined the function

$$
\begin{aligned}
R(z) & =\int_{0}^{1} \frac{d q}{q^{2}}(1-q)\left|e^{-i q z}-1\right|^{2} \\
& =-2\left(1+\gamma_{E}+\ln z-\cos z-\mathrm{Ci} z-z \mathrm{Si} z\right),
\end{aligned}
$$

$\mathrm{Si}$ and $\mathrm{Ci}$ are the sine and cosine integral functions, and $\gamma_{E} \approx$ 0.577216 is the Euler constant.

\section{Resulting long-time momentum distribution}

It is also interesting to discuss the steady-state momentum distribution within this framework, especially in the regime $p(1-p) \ll 1$ where deviations from thermal behavior are largest. As a first step we use Eq. (45) to derive a form for the real-space correlation function at large $|x|$ that includes subleading terms omitted from Eq. (37), obtaining

$$
\mathcal{G}(x) \approx \frac{i}{2 \pi} \frac{\left|e^{1+\gamma_{E}} Q x\right|^{2 p(1-p)}}{x+i a} e^{-\pi p(1-p)|Q x|} .
$$

From this, using Eq. (11), we find that the stationary momentum distribution for $|k| \ll Q$ is

$$
n(k, t)=\frac{1}{2}-\frac{1}{\pi} e^{\alpha\left(1+\gamma_{E}\right)} \Gamma(\alpha) \operatorname{Im}\left[(-i k / Q+\pi \alpha / 2)^{-\alpha}\right],
$$

where $\alpha \equiv 2 p(1-p)$. A striking point is that two scales appear in Eq. (48): one is $Q$, while the second one is $\pi \alpha Q / 2$. These scales are widely separated when $\alpha$ is small, and so there are two distinct small $k$ regimes of behavior for $n(k, t)$ at long times, with

$$
n(k, t)=\frac{1}{2}-C(\pi \alpha / 2)^{-(1+\alpha)} \alpha k / Q
$$

for $k \ll \pi \alpha Q / 2$ and

$$
n(k, t)=\frac{1}{2}-C(\pi \alpha / 2)[1-\alpha Q / k](Q / k)^{\alpha}
$$

for $\pi \alpha Q / 2 \ll k \ll Q$, where $C=\frac{1}{\pi} e^{\alpha\left(1+\gamma_{E}\right)} \Gamma(\alpha)$.

As a supplement to this discussion of asymptotic forms, the full momentum distribution at long times can be obtained numerically from Eqs. (45) and (11). We present its behavior in Fig. 2. Since the quantity measured experimentally is a derivative, we plot the difference in derivatives between the steady-state distribution and the one for a thermal state with the same energy density. As anticipated in our earlier discussion, these differences are largest when $p(1-p)$ is small.

\section{EQUILIBRATION AT $\boldsymbol{v}=2$}

In this section we study relaxation of a nonequilibrium distribution in a system of two channels coupled by contact interactions. Whereas contact interactions at $v=1$ simply renormalize the Fermi velocity, without providing a mechanism for relaxation, at $v=2$ they give rise to two linearly dispersing collective modes with distinct velocities, and hence do produce relaxation. We therefore focus on this as the simplest interesting case. We discuss briefly the effects of finite-range interactions at $v=2$ in Sec. V C 3 .

The Hamiltonian of the system is given by Eq. (6), with $n_{c}=2$ and an interchannel interaction potential

$$
U_{12}\left(x-x^{\prime}\right)=U_{21}\left(x-x^{\prime}\right)=(g / 2) \delta\left(x-x^{\prime}\right) .
$$

Intrachannel interactions are absorbed into the Fermi velocities $v_{1,2}$.

We start this section with a general discussion of time evolution for this Hamiltonian. Then we set out the solution to a special case, calculating the electron correlation function at arbitrary time for a particular choice of initial state, in which each channel is separately in thermal equilibrium but at different temperatures. Finally, we extend the approach described in Sec. IV to calculate the long-time asymptotics of the correlation function at long and short distances for an arbitrary initial single-particle distribution.

\section{A. Diagonalization of bosonic Hamiltonian}

The bosonized form of the Hamiltonian is

$$
\begin{aligned}
\hat{H}= & \hbar v_{1} \sum_{k>0} k \hat{a}_{k}^{+} \hat{a}_{k}+\hbar v_{2} \sum_{k>0} k \hat{b}_{k}^{+} \hat{b}_{k} \\
& +\frac{1}{2} \frac{g}{2 \pi} \sum_{k>0} k\left(\hat{a}_{k} \hat{b}_{k}^{+}+\hat{a}_{k}^{+} \hat{b}_{k}\right),
\end{aligned}
$$

where $\hat{a}_{k}$ and $\hat{b}_{k}$ are the boson operators on the first and the second channel. It can be diagonalized by the rotation

$$
\left(\hat{\alpha}_{k}, \hat{\beta}_{k}\right)^{T}=\mathrm{S}\left(\hat{a}_{k}, \hat{b}_{k}\right)^{T},
$$

with

$$
\mathrm{S}=\left(\begin{array}{cc}
\cos \theta & \sin \theta \\
-\sin \theta & \cos \theta
\end{array}\right)
$$

Setting $\gamma=g /(2 \pi \hbar)$ and $\tan 2 \theta=\gamma /\left(v_{1}-v_{2}\right)$, this brings the Hamiltonian to the diagonal form

$$
\hat{H}=\hbar v_{+} \sum_{k>0} k \hat{\alpha}_{k}^{+} \hat{\alpha}_{k}+\hbar v_{-} \sum_{k>0} k \hat{\beta}_{k}^{+} \hat{\beta}_{k} .
$$

The collective mode velocities $v_{ \pm}$are

$$
\begin{aligned}
& v_{+}=v_{1} \cos ^{2} \theta+v_{2} \sin ^{2} \theta+\frac{1}{2} \gamma \sin 2 \theta, \\
& v_{-}=v_{1} \sin ^{2} \theta+v_{2} \cos ^{2} \theta-\frac{1}{2} \gamma \sin 2 \theta .
\end{aligned}
$$

The time dependence of the operators $\hat{\alpha_{k}}$ and $\hat{\beta_{k}}$ in the Heisenberg representation is

$$
\hat{\alpha}_{k}(t)=e^{-i v_{+} k t} \hat{\alpha}_{k}, \quad \hat{\beta}_{k}(t)=e^{-i v_{-} k t} \hat{\beta}_{k} .
$$

Since we wish to discuss time evolution from an initial state specified by occupation numbers for independent, noninteracting fermions, we need to re-express Eq. (55) in terms of the operators $\hat{a}$ and $\hat{b}$ in their Schrödinger representation, using 
the transformation $\mathrm{S}$ and its inverse. In this way we obtain

$$
\begin{aligned}
\hat{a}_{k}(t) & =\mathfrak{u}_{k}(t) \hat{a}_{k}+\mathfrak{s}_{k}(t) \hat{b}_{k} \\
\text { and } \quad \hat{b}_{k}(t) & =\mathfrak{s}_{k}(t) \hat{a}_{k}+\mathfrak{v}_{k}(t) \hat{b}_{k} .
\end{aligned}
$$

with coefficients

$$
\begin{aligned}
\mathfrak{u}_{k}(t) & =e^{-i v_{+} k t} \cos ^{2} \theta+e^{-i v_{-} k t} \sin ^{2} \theta, \\
& \mathfrak{v}_{k}(t)=e^{-i v_{+} k t} \sin ^{2} \theta+e^{-i v_{-} k t} \cos ^{2} \theta, \\
\text { and } \quad \mathfrak{s}_{k}(t) & =\left(e^{-i v_{+} k t}-e^{-i v_{-} k t}\right) \cos \theta \sin \theta .
\end{aligned}
$$

Equations (56) and (57) define a unitary transformation of the boson operators that generates their time evolution independently for each wave vector.

In bosonized form, the electron correlation function is

$$
\begin{aligned}
G_{\eta}(x, t) & =\left\langle e^{i \hat{H} t} \hat{\psi}_{\eta}^{+}(x) \hat{\psi}_{\eta}(0) e^{-i \hat{H} t}\right\rangle \\
& =(2 \pi a)^{-1}\left\langle e^{i \hat{\phi}_{\eta}(x, t)} e^{-i \hat{\phi}_{\eta}(0, t)}\right\rangle .
\end{aligned}
$$

For an initial state without correlations between the channels, this factorizes into the product

$$
G_{\eta}(x, t)=(2 \pi a)^{-1} G_{a}^{(\eta)}(x, t) G_{b}^{(\eta)}(x, t),
$$

where

$$
G_{a, b}^{(\eta)}(x, t)=\left\langle e^{i \hat{\phi}_{a, b}^{(\eta)}(x, t)} e^{-i \phi_{a, b}^{(\eta)}(0, t)}\right\rangle
$$

and [from Eq. (56)]

$$
\hat{\phi}_{a}^{(1)}(x, t)=-\sum_{q>0}(2 \pi / q L)^{1 / 2}\left(\mathfrak{u}_{q}(t) \hat{a}_{q} e^{i q x}+\text { H.c. }\right) e^{-q a / 2} .
$$

The corresponding expression for $\hat{\phi}_{b}^{(1)}(x, t)$ is obtained by making the substitutions $\mathfrak{u}_{q}(t) \rightarrow \mathfrak{s}_{q}(t)$ and $\hat{a}_{q} \rightarrow \hat{b}_{q}$ in Eq. (60). Similarly, we obtain an expression for bosonic fields in the second channel by making the substitutions $\mathfrak{s}_{q} \rightarrow \mathfrak{v}_{q}$ and $\mathfrak{u}_{q} \rightarrow \mathfrak{s}_{q}$ in the equations for $\hat{\phi}_{a, b}^{(1)}(x, t)$.

\section{B. Equilibration of channels with unequal initial temperatures}

We next study relaxation from an initial state in which the particles in channels of the system without interactions have thermal distributions with different temperatures, $T_{\eta}$. A similar problem was studied in a context of a quantum quench for a nonchiral Luttinger liquid in Ref. 24.

The simplifying feature of a thermal initial state is that there is uncorrelated occupation of both the fermion orbitals on which $\hat{c}_{k \eta}$ act and the boson orbitals on which $\hat{a}_{k}$ and $\hat{b}_{k}$ act. We can therefore specify this state by the averages

$$
\left\langle\hat{a}_{k}^{+} \hat{a}_{k}\right\rangle=\left(e^{\hbar v_{1} k \beta_{1}}-1\right)^{-1}, \quad\left\langle\hat{b}_{k}^{+} \hat{b}_{k}\right\rangle=\left(e^{\hbar v_{2} k \beta_{2}}-1\right)^{-1},
$$

where $\beta_{\eta}=1 / k_{B} T_{\eta}$. Correlators of the fields $\hat{\phi}_{a, b}^{(\eta)}(x, t)$ are those for a noninteracting edge, with the appropriate choice for velocity. We use the notation $w_{\eta}=-2 i \beta_{\eta} \hbar v_{\eta} / L$ and $\xi_{\eta}=$ $\pi / \beta_{\eta} \hbar v_{\eta}$. We also define $r=\frac{1}{2} \sin ^{2} 2 \theta, s=1-r$, and

$$
v_{D}=v_{+}-v_{-}=\frac{\gamma}{\sin 2 \theta} \text {. }
$$

In this way we obtain

$$
\begin{aligned}
G_{a}^{(1)}(x, t) & \\
= & (2 \pi a / L)^{s} \frac{1}{w_{1}^{s}} \frac{1}{\sinh ^{s}\left[\xi_{1}(x+i a)\right]} \\
& \times\left[\frac{\sinh \left[\xi_{1}\left(v_{D} t+i a\right)\right] \sinh \left[\xi_{1}\left(-v_{D} t+i a\right)\right]}{\sinh \left[\xi_{1}\left(x+v_{D} t+i a\right)\right] \sinh \left[\xi_{1}\left(x-v_{D} t+i a\right)\right]}\right]^{r / 2} .
\end{aligned}
$$

In a similar way we get for $G_{b}^{(1)}(x, t)$

$$
\begin{aligned}
G_{b}^{(1)}(x, t) & \\
= & (2 \pi a / L)^{r} \frac{1}{w_{2}^{r}} \frac{1}{\sinh ^{r}\left[\xi_{2}(x+i a)\right]} \\
& \times\left[\frac{\sinh \left[\xi_{2}\left(x+v_{D} t+i a\right)\right] \sinh \left[\xi_{2}\left(x-v_{D} t+i a\right)\right]}{\sinh \left[\xi_{2}\left(v_{D} t+i a\right)\right] \sinh \left[\xi_{2}\left(-v_{D} t+i a\right)\right]}\right]^{r / 2} .
\end{aligned}
$$

As a check, note that the initial electron correlation function

$$
\left\langle\hat{G}_{\eta}(x, 0)\right\rangle=(i / 2 \pi) \xi_{\eta} \sinh ^{-1}\left[\xi_{\eta}(x+i a)\right]
$$

derived in this way from Eq. (59) coincides with the thermal one at inverse temperature $\beta_{\eta}$. Next consider the long-time asymptotics of $G_{\eta}(x, t)$. The function $1 / \sinh \left[\xi_{\eta}(x+i a)\right]$ is peaked near $x=0$ and falls off exponentially at large $x$ on a scale $1 / \xi_{\eta}$ (or as a power-law at zero temperature). For a fixed $x$ at long times, the factor $[\cdots]^{r / 2}$ approaches 1 . The length scale defined by the product $\sinh \left[\xi_{1}(x+i a)\right]^{-s} \sinh \left[\xi_{2}(x+\right.$ $i a)]^{-r}$ is

$$
l=\left(s \xi_{1}+r \xi_{2}\right)^{-1}=\frac{\hbar}{\pi}\left[\frac{s}{\beta_{1} v_{1}}+\frac{r}{\beta_{2} v_{2}}\right]^{-1} .
$$

The time taken to approach the asymptotic form is $\tau \approx l / v_{D}$. For times much longer than $\tau$ we have

$$
\begin{aligned}
G_{1}(x)= & \frac{i}{2 \hbar} \frac{1}{\left(\beta_{1} v_{1}\right)^{s}} \frac{1}{\sinh ^{s}\left[\xi_{1}(x+i a)\right]} \\
& \times \frac{1}{\left(\beta_{2} v_{2}\right)^{r}} \frac{1}{\sinh ^{r}\left[\xi_{2}(x+i a)\right]} .
\end{aligned}
$$

We see that at long times the system reaches a steady state. In this state the electron correlation function differs strikingly from a thermal one, being given by a product of two thermal correlation functions for systems at different temperatures, each raised to powers that depend on the strength of the interchannel interactions. Comparison of electron momentum distribution obtained from Eq. (65) with a thermal one is presented in Fig. 3.

Clearly, an attraction of studying evolution from this special class of initial states is that one obtains explicit results not only at long times, but at all intermediate times. In Fig. 4 we exploit this to show the time evolution of the momentum distribution in a channel initially at zero temperature coupled to a second channel with nonzero initial temperature.

\section{Correlations at $\boldsymbol{v}=\mathbf{2}$ in terms of initial density operator}

As a second example of relaxation in the two-channel system, we consider an initial state with a double step distribution of electron momentum in one channel. The correlation 


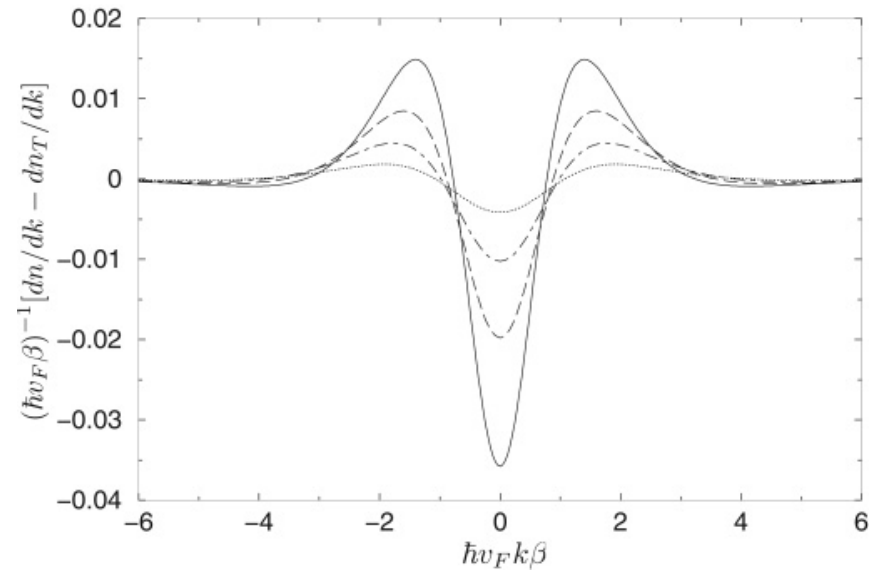

FIG. 3. Difference between derivatives of the electron momentum distribution functions in a steady state described by Eq. (65) with $T_{2}^{*}=0$ and in a thermal state at temperature $T=\sqrt{s} T_{1}^{*}$; (thick solid line) $\theta=\pi / 4$; (dashed line) $\theta=\pi / 6$; (dot-dashed line) $\theta=\pi / 8$; (dotted line) $\theta=\pi / 12$.

function decouples into a product [Eq. (59)] of factors that can each be expressed using the approach of Sec. IV A in terms of the initial electron density operator. For example,

$$
G_{a}^{(1)}(x, t)=e^{\frac{1}{2}\left[\hat{\phi}_{a}(x, t), \hat{\phi}_{a}\left(x^{\prime}, t\right)\right]}\left\langle e^{i \int K\left(x, x^{\prime}, t ; y\right) \hat{\rho}(y) d y}\right\rangle,
$$

where the kernel is given by

$K\left(x, x^{\prime}, t ; y\right)=-i \sum_{q \neq 0}(2 \pi / q L) \mathfrak{u}_{k}^{*}(t)\left(e^{-i q x}-e^{-i q x^{\prime}}\right) e^{i q y-|q| a}$

and has the form

$$
\begin{aligned}
& K\left(x, x^{\prime}, t ; y\right) \\
& =\pi\left(\left[\operatorname{sgn}\left(v_{+} t-x+y\right)-\operatorname{sgn}\left(v_{+} t-x^{\prime}+y\right)\right] \cos ^{2} \theta\right. \\
& \left.\quad+\left[\operatorname{sgn}\left(v_{-} t-x+y\right)-\operatorname{sgn}\left(v_{-} t-x^{\prime}+y\right)\right] \sin ^{2} \theta\right) .
\end{aligned}
$$

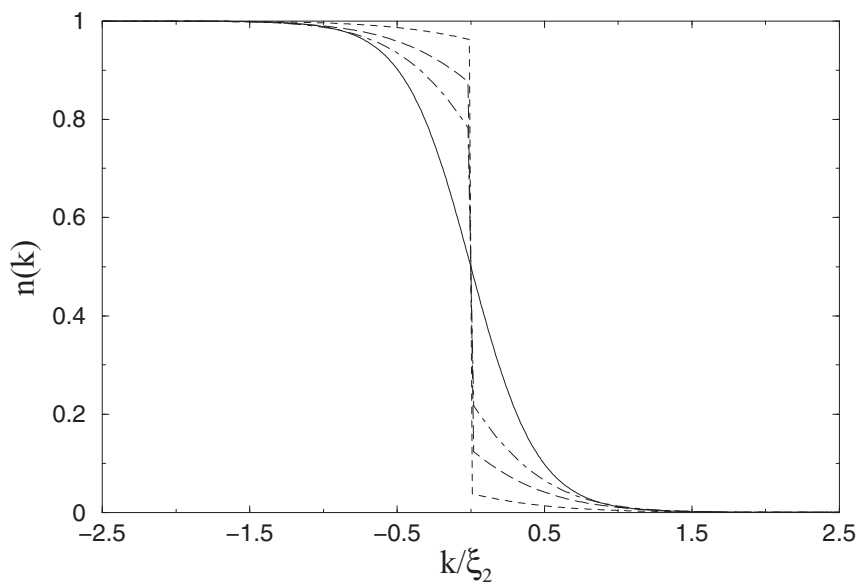

FIG. 4. Time evolution of the electron momentum distribution for a system of two channels in the strong-couping limit $(\theta=\pi / 4)$ with unequal initial temperatures $T_{1}^{*}=0$ and $T_{2}^{*}$, obtained using Eqs. (59), (63), and (64); (dotted line) $\xi_{2} v_{D} t=1$; (dashed line) $\xi_{2} v_{D} t=2$; (dot-dashed line) $\xi_{2} v_{D} t=3$; (solid line) thermal distribution at temperature $T=T_{2}^{*} / \sqrt{2}$, shown for comparison.
At times $t>\left|\left(x-x^{\prime}\right) / v_{D}\right|$ this kernel separates into two steps: one occupying the interval $\left(x^{\prime}-v_{+} t, x-v_{+} t\right)$ and the other in $\left(x^{\prime}-v_{-} t, x-v_{-} t\right)$. The separation between these intervals grows at speed $v_{D} t$. An energy scale $\mathrm{eV}$ in the initial state sets a characteristic length $\hbar v / e V$ : the time scale $\tau$ introduced in Sec. II is the time taken for the separation between steps to reach this characteristic length. Introducing an operator

$$
\hat{N}(x)=\int_{0}^{x} \hat{\rho}(z) d z
$$

that counts the number of particles in an interval of length $x$, in the long-time limit there is the factorization

$$
\left\langle e^{i \int K(x, 0, t ; y) \hat{\rho}(y) d y}\right\rangle=\left\langle e^{-2 i \pi \hat{N}(x) \cos ^{2} \theta}\right\rangle\left\langle e^{-2 i \pi \hat{N}(x) \sin ^{2} \theta}\right\rangle .
$$

Here we see a contrast between our earlier results at $v=1$ in systems with finite range or Coulomb interactions, for which $\omega_{q}$ has dispersion, and these results at $v=2$ with contact interactions between electrons in different channels, for which the bosonic modes have linear dispersion relations. While in the former case the steady-state correlation function can be expressed solely in terms of the two-point density correlation function of the initial state [see Eq. (30)], this is not so in the latter case. Nevertheless, simplifications arise in the small and large $x$ asymptotics of the correlation function, as we now show.

\section{Short-distance asymptotics}

The short-distance form of the steady-state correlation function is fixed by the energy density in each channel in the initial state, characterized by effective temperatures $T_{\eta}^{*}$. Setting $\xi_{\eta}^{*}=\pi k_{B} T_{\eta}^{*} / \hbar v_{\eta}$, we find

$$
G_{1}(x)=\frac{i}{2 \pi} \frac{1}{x+i a}\left[1+\frac{x^{2}}{3 !}\left(s \xi_{1}^{* 2}+r \xi_{2}^{* 2}\right)+\cdots\right]^{-1} .
$$

Correlations at short distances in channel 1 therefore have an effective temperature

$$
T_{\mathrm{eff}}^{2}=s T_{1}^{* 2}+r T_{2}^{* 2},
$$

with the corresponding result for channel 2 obtained by exchanging $r$ and $s$. We see that the effective temperatures of the two channels differ, except in the strong-coupling limit where $r=s=1 / 2$.

\section{Large-distance asymptotics}

Calculation of the large $x$ asymptotics of the Green function in the form given by exponent of the electron counting operator is a well-studied problem. The asymptotics can be obtained using the theory of Toeplitz determinants; see, for example, Ref. 12

In the case of a double-step distribution of width $Q$, which is created and measured in channel 1 with channel 2 initially at zero temperature, we obtain for $Q|x| \gg 1$ in the leading order

$$
G_{1}(x) \approx \frac{i}{2 \pi} \frac{1}{x+i a} \exp \left[-\bar{s} \frac{|Q x|}{2 \pi}\right],
$$

where

$$
\begin{aligned}
\bar{s}= & -\frac{1}{2}\left[\ln \left(1-4 p(1-p) \sin ^{2}\left[\pi \cos ^{2} \theta\right]\right)\right. \\
& \left.+\ln \left(1-4 p(1-p) \sin ^{2}\left[\pi \sin ^{2} \theta\right]\right)\right] ;
\end{aligned}
$$


see Refs. 12 and 25 for details.

\section{Finite-range interactions at $v=2$}

Finite-range interactions, in contrast to contact interactions, generate dispersion so the mode velocities $v_{ \pm}$and the mixing angle $\theta$ become functions of wave vector. Dispersion in the two mode velocities provides a further mechanism for relaxation at $v=2$. One sees, however, from Eqs. (1) and (2) that this additional mechanism is less important at small $V$ than the one due to contact interactions, since it yields a much longer relaxation time (scaling as $V^{-3}$ in place of $V^{-1}$ ). Thus at small $V$ there is window between these two time scales during which the results for contact interactions apply even in the presence of dispersion. At times beyond the longer scale, our results for $v=1$ carry over with simple modifications and we obtain for $t \rightarrow \infty$

$$
G_{1}(x)=\left[\mathcal{G}_{a}(x)\right]^{\cos ^{4} \theta+\sin ^{4} \theta}\left[\mathcal{G}_{b}(x)\right]^{2 \cos ^{2} \theta \sin ^{2} \theta} .
$$

Here $\mathcal{G}_{a}(x)$ and $\mathcal{G}_{b}(x)$ are obtained from the expression for $\mathcal{G}(x)$ in Eq. (30) by computing expectations values in the initial stares of channel 1 and 2 , respectively, while $\theta$ denotes the limiting value of the mixing angle at small vectors.

\section{TUNNELING DENSITY OF STATES}

As summarized in Sec. II, the experiments of Refs. 5-7 probe the electron distribution by means of tunneling measurements. With that as a motivation, we study in this section the tunneling density of states (TDOS). We consider in particular edge channels at $v=2$ after long-time evolution from an initial state characterized by two temperatures, as introduced in Sec. V B. We focus on this case rather than an initial state with a double-step distribution that would better represent the experimental situation, because the analytic results available for the Green function with these initial conditions facilitate accurate evaluation of the TDOS. It is important to stress that, while for a noninteracting system the electron momentum distribution and the tunneling density of states are simply related, with interactions they become independent quantities. This is illustrated (in the space and time rather than momentum and energy domains) in our results below by contrasting Eq. (65) with Eq. (73).

For a single edge state in equilibrium at temperature $T$ with only contact interactions, the TDOS defined in Eq. (14) has the form

$$
v(E)=(\hbar v)^{-1} n_{F}\left(E / k_{B} T\right),
$$

and so the tunneling current is proportional to the Fermi distribution $n_{F}(x)=\left(e^{x}+1\right)^{-1}$. More generally, one can attempt to characterize the energy dependence of the TDOS by an effective temperature $T_{\text {eff }}$. As there is no unique way to do so, we explore two alternatives. First, since the energy density in thermal equilibrium is $\frac{\pi}{12}\left(k_{\mathrm{B}} T\right)^{2}$, one natural definition of $T_{\text {eff }}$ is via

$$
\frac{\pi}{12}\left(k_{B} T_{\mathrm{eff}}\right)^{2}=\left(2 \pi v_{\mathrm{eff}}\right)^{-1} \int_{-\infty}^{\infty}\left[v(E)-v_{\mathrm{eff}} \theta(-E)\right] E d E,
$$

where $\theta(E)$ is the Heaviside step function and the normalization is $v_{\text {eff }} \equiv v(-\infty)$, the TDOS far from the Fermi energy. To evaluate $v(E)$ we require [see Eq. (14)] the correlator

$$
G_{\eta}(\tau)=\lim _{t \rightarrow \infty}\left\langle\hat{\psi}^{+}(x, t+\tau) \psi(x, t)\right\rangle .
$$

Consider the two-channel problem discussed in Sec. V. In thermal equilibrium the correlator $G_{1}(t)$ reads

$$
\begin{aligned}
G_{1}(t)= & \frac{i}{2 \beta v_{+}^{\cos ^{2} \theta} v_{-}^{\sin ^{2} \theta}} \frac{1}{\sinh \left[\frac{\pi}{\beta v_{+}}\left(-v_{+} t+i a\right)\right]^{\cos ^{2} \theta}} \\
& \times \frac{1}{\sinh \left[\frac{\pi}{\beta v_{-}}\left(-v_{-} t+i a\right)\right]^{\sin ^{2} \theta}},
\end{aligned}
$$

which gives for the density of states

$$
v(E)=\left(\hbar v_{+}^{\cos ^{2} \theta} v_{-}^{\sin ^{2} \theta}\right)^{-1} n_{F}\left(E / k_{B} T\right) .
$$

Thus the equilibrium TDOS in a two channel system with contact interactions between electrons in each channel is proportional to the Fermi distribution and independent of interaction strength. It is worth noting that this is not the case for a single channel with finite-range or Coulomb interactions.

Now let us consider the tunneling density of states at long times, when the system starts out of equilibrium. In order to have explicit results that are easy to evaluate accurately for all energies, we consider an initial state with channels at unequal temperatures $T_{1,2}^{*}$. For this case the Green function in channel " 1 " at long times reads

$$
\begin{aligned}
G_{1}(t)= & L^{-1} w_{1}^{-s} w_{2}^{-r} \\
& \times \frac{1}{\sinh \left[\xi_{2}\left(-v_{+} t+i a\right)\right]^{r / 2} \sinh \left[\xi_{2}\left(-v_{-} t+i a\right)\right]^{r / 2}} \\
& \times \frac{1}{\sinh \left[\xi_{1}\left(-v_{+} t+i a\right)\right]^{\cos ^{4} \theta} \sinh \left[\xi_{1}\left(-v_{-} t+i a\right)\right]^{\sin ^{4} \theta}} .
\end{aligned}
$$

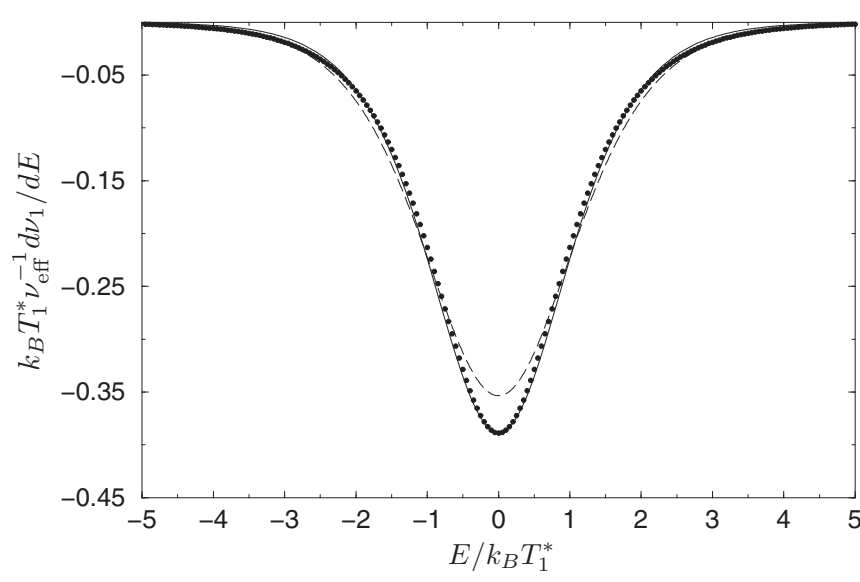

FIG. 5. Derivative of the normalized tunneling density of states as a function of energy in a two-channel system. (Solid circles) In the steady state reached with initial temperatures $T_{1}^{*}>0$ and $T_{2}^{*}=0$ at $g / \hbar v_{1}=1$ in the strong coupling limit $v_{1}=v_{2}$. (Dashed line) In a thermal state at the temperature $T_{1}^{*} / \sqrt{2}$ expected from equipartition. (Solid line) In a thermal state with a temperature $0.64 \times T_{1}^{*}$ chosen to give the best fit to the filled circles. 
This gives for the integral Eq. (72)

$$
\begin{aligned}
\frac{\pi}{12}\left(k_{B} T_{\mathrm{eff}}\right)^{2}= & \frac{\pi\left(k_{B} T_{1}^{*}\right)^{2}}{24 v_{1}^{2}}\left[\left(v_{+}^{2}-v_{-}^{2}\right) \cos 2 \theta\right. \\
& \left.+\left(v_{+}^{2}+v_{-}^{2}\right)\left\{s+\left(v_{1} T_{2}^{*} / v_{2} T_{1}^{*}\right)^{2} r\right\}\right] .
\end{aligned}
$$

The effective temperature $T_{\text {eff }}$ obtained from Eq. (74) is a function of two parameters: $\theta$ and $v_{2} / v_{1}$. For $v_{1}=v_{2}$ and with the second channel at an initial temperature $T_{2}^{*}=0$ we obtain

$$
\frac{\pi}{12}\left(k_{B} T_{\text {eff }}\right)^{2}=\frac{\pi}{24}\left(k_{B} T_{1}^{*}\right)^{2}\left[1+\left(\gamma / 2 v_{1}\right)^{2}\right] .
$$

From this we find $T_{\text {eff }}=\left(T_{1}^{*} / \sqrt{2}\right)\left[1+\left(\gamma / 2 v_{1}\right)^{2}\right]^{1 / 2}$, which is always higher than the equipartition value $T_{1}^{*} / \sqrt{2}$.

An alternative way to define $T_{\text {eff }}$ is to fit the normalized TDOS to a thermal Fermi function, as displayed in Fig. 5. Interestingly, with $v_{1}=v_{2}$ and for $g / \hbar v_{1} \lesssim 1$, the best fit yields $T_{\text {eff }} \sim 0.64 \times T_{1}^{*}$, which is about $9 \%$ lower than the equipartition result.

We believe these calculations of effective temperature make some useful points. First, they show that there is no unique definition of effective temperature because the steady state is not thermal. Second, they serve to demonstrate that it is possible for effective temperature (at least, by our second definition and for the initial state we have treated) to be lower than expected from equipartition. Further, the specific mechanism for a low effective temperature is clear: the derivative of the tunneling density of states at long times has less weight in its peak and more weight in its flanks than in thermal equilibrium.

In the experiments of Refs. 5 and 6 an apparent energy loss is reported from analysis of the TDOS far from the QPC, compared to behavior close to the QPC. The experimental analysis uses two methods to find an effective temperature, corresponding to the two definitions we have employed. Although no systematic discrepancies are reported between results from the two methods, the first method (calculation of energy density by integration) relies on capturing contributions from the flanks of the distribution, which in turn requires an accurate value for the baseline. It may, therefore, be less robust than the second method (fitting to a Fermi function). We find it interesting to note that the temperature reduction we obtain from this method is within the error bars of the results found in Ref. 6.

\section{ACKNOWLEDGMENTS}

We acknowledge discussions with L. Glazman, Y. Gefen, A. Mirlin, D. Mailly, and F. Pierre. This work was supported by EPSRC under Grant No. EP/D050952/1.
${ }^{1}$ T. Kinoshita, T. Wenger, and D. S. Weiss, Nature 440, 900 (2006). ${ }^{2}$ S. Hofferberth, I. Lesanovsky, B. Fischer, T. Schumm, and J. Schmiedmayer, Nature 449, 324 (2007).

${ }^{3}$ I. Neder, M. Heiblum, Y. Levinson, D. Mahalu, and V. Umansky, Phys. Rev. Lett. 96, 016804 (2006).

${ }^{4}$ G. Granger, J. P. Eisenstein, and J. L. Reno, Phys. Rev. Lett. 102, 086803 (2009).

${ }^{5}$ C. Altimiras, H. le Sueur, U. Gennser, A. Cavanna, D. Mailly, and F. Pierre, Nat. Phys. 6, 34 (2009).

${ }^{6}$ H. le Sueur, C. Altimiras, U. Gennser, A. Cavanna, D. Mailly, and F. Pierre, Phys. Rev. Lett. 105, 056803 (2010).

${ }^{7}$ C. Altimiras, H. le Sueur, U. Gennser, A. Cavanna, D. Mailly, and F. Pierre, Phys. Rev. Lett. 105, 226804 (2010).

${ }^{8}$ S. Wall et al., Nat. Phys. 7, 114 (2011).

${ }^{9}$ P. Calabrese and J. Cardy, J. Stat. Mech. (2007) P06008; Phys. Rev. Lett. 96, 136801 (2006).

${ }^{10}$ M. Rigol, V. Dunjko, and M. Olshanii, Nature 452, 854 (2008).

${ }^{11}$ M. Rigol, V. Dunjko, V. Yurovsky, and M. Olshanii, Phys. Rev. Lett. 98, 050405 (2007); C. Kollath, A. M. Läuchli, and E. Altman, ibid. 98, 180601 (2007); S. R. Manmana, S. Wessel, R. M. Noack, and A. Muramatsu, ibid. 98, 210405 (2007).

${ }^{12}$ D. B. Gutman, Y. Gefen, and A. D. Mirlin, Phys. Rev. B 81, 085436 (2010); J. Phys. A: Math. Theor. 44, 165003 (2011).

${ }^{13}$ X. G. Wen, Phys. Rev. Lett. 64, 2206 (1990); Phys. Rev. B 43, 11025 (1991).
${ }^{14}$ A. M. Lunde, S. E. Nigg, and Markus Büttiker, Phys. Rev. B 81, 041311(R) (2010).

${ }^{15}$ P. Degiovanni, C. Grenier, G. Fève, C. Altimiras, H. le Sueur, and F. Pierre, Phys. Rev. B 81, 121302(R) (2010).

${ }^{16}$ J. T. Chalker, Yuval Gefen, and M. Y. Veillette, Phys. Rev. B 76, 085320 (2007).

${ }^{17}$ I. P. Levkivskyi and E. V. Sukhorukov, Phys. Rev. B 78, 045322 (2008).

${ }^{18}$ D. L. Kovrizhin and J. T. Chalker, Phys. Rev. B 80, 161306(R) (2009); 81, 155318 (2010).

${ }^{19}$ Using the notation of Ref. 15, the quantity we denote by $\tau$ in Eq. (1) is $L_{V} / v_{0}$.

${ }^{20}$ T. Otsuka, E. Abe, Y. Iye, and S. Katsumoto, Phys. Rev. B 81, 245302 (2010)

${ }^{21}$ See, for example, H. Bruus and K. Flensberg, Many-Body Quantum Theory in Condensed Matter Physics (Oxford University Press, Oxford, UK, 2004); G. D. Mahan, Many-Particle Physics (Kluwer Academic/Plenum, New York, 2000).

${ }^{22}$ See J. von Delft and H. Schoeller, Ann. Phys. 7, 225 (1998); T. Giamarchi, Quantum Physics in One Dimension (Oxford University Press, Oxford, UK, 2004).

${ }^{23}$ I. E. Dzyaloshinskii and A. I. Larkin, Sov. Phys. JETP 38, 202 (1974).

${ }^{24}$ M. A. Cazalilla, Phys. Rev. Lett. 97, 156403 (2006); A. Iucci and M. A. Cazalilla, Phys. Rev. A 80, 063619 (2009).

${ }^{25}$ D. L. Kovrizhin and J. T. Chalker (paper in preparation). 